\title{
AN EXPLORATION OF SPATIAL-SPECTRAL INFORMATION USING HYPERSPECTRAL IMAGE ENHANCEMENT
}

\author{
Nagarajan Munusamy ${ }^{1}$ and Rashmi. P. Karchi2 ${ }^{2}$ \\ ${ }^{1}$ Department of Computer Science (Multimedia and Web Technology), \\ KSG College of Arts and Science, \\ Coimbatore - 641 015, Tamilnadu, India \\ $2^{2 *}$ Department of Computer Science, Bharathiar University, \\ Coimbatore-641046, Tamilnadu, India \\ ${ }^{1}$ mnaagarajan@gmail.com, ${ }^{2 *}$ rashmikarchi@gmail.com
}

\begin{abstract}
Spectral imaging widens the capabilities about studying qualitative and quantitative features. Spectral imaging is a new advantageous tool that merges two scientific methodologies, namely spectroscopy and imaging. It is important to measure the spectrum at each point of the image that entails combining dispersive optics with the more common imaging tools, and introduces constrains as well. The hyperspectral imagery of the Mars dataset contains abundant spatial and spectral information. To identify the mineral composition on the surface of the Mars hyperspectral imagery by performing the enhancement, end- member detection or extraction and unmixing. Spectral imaging analysis is very much applicable in analyzing the complex data structure which cannot be analyzed visually. This paper present hyperspectral image enhancement techniques by employing the different filters/kernels in-order to explore the embedded spatial-spectral information. In this paper few of the algorithms and examples are discussed with prominence on the practice for different experimental manners (fluorescence and bright field). Spectral imaging analysis is done with considering hyperspectral imagery using Environmental(ENVI) tool. On the other hand, several applications have already shown its potential. The experiment produce the promising results when the Mars image is enhanced by using the Median and Gaussian filters. Further the mineral composition identification is to be performed from the enhanced Mars surface hyperspectral imagery.
\end{abstract}

Keywords - End-member, Enhancement, ENVI Tool, Hyperspectral Imagery, Image Analysis, Spectral Unmixing, Spectroscopy

\section{INTRODUCTION}

Spectroscopy is nothing but the Reflectance spectroscopy that focuses on study of the electromagnetic spectrum reflected by various materials. The spectroscopy is learning of light that can be produced by or reflected as of resources and its difference in energy by wavelength. Electromagnetic radiation consists the more energetic the light when there is the shorter the wavelength of energy. In hyperspectral imagery it is a task to extract the minerals present in the soil or rock of a Martian surface. Then to find the composition of

Received: March 19, 2019

Reviewed: July 17, 2019

Accepted: March 11, 2020

* Corresponding Author

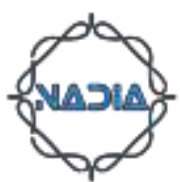


minerals on the surface of the Mars it is necessary to read their wavelength and bandwidth. According to Electromagnetic radiation theory rocks and soil can absorb radiation at certain energy levels and bears their respective wavelengths of radiation. The respective wavelength radiation allows the scientists to identify them through their spectra as shown in the Figure 1.

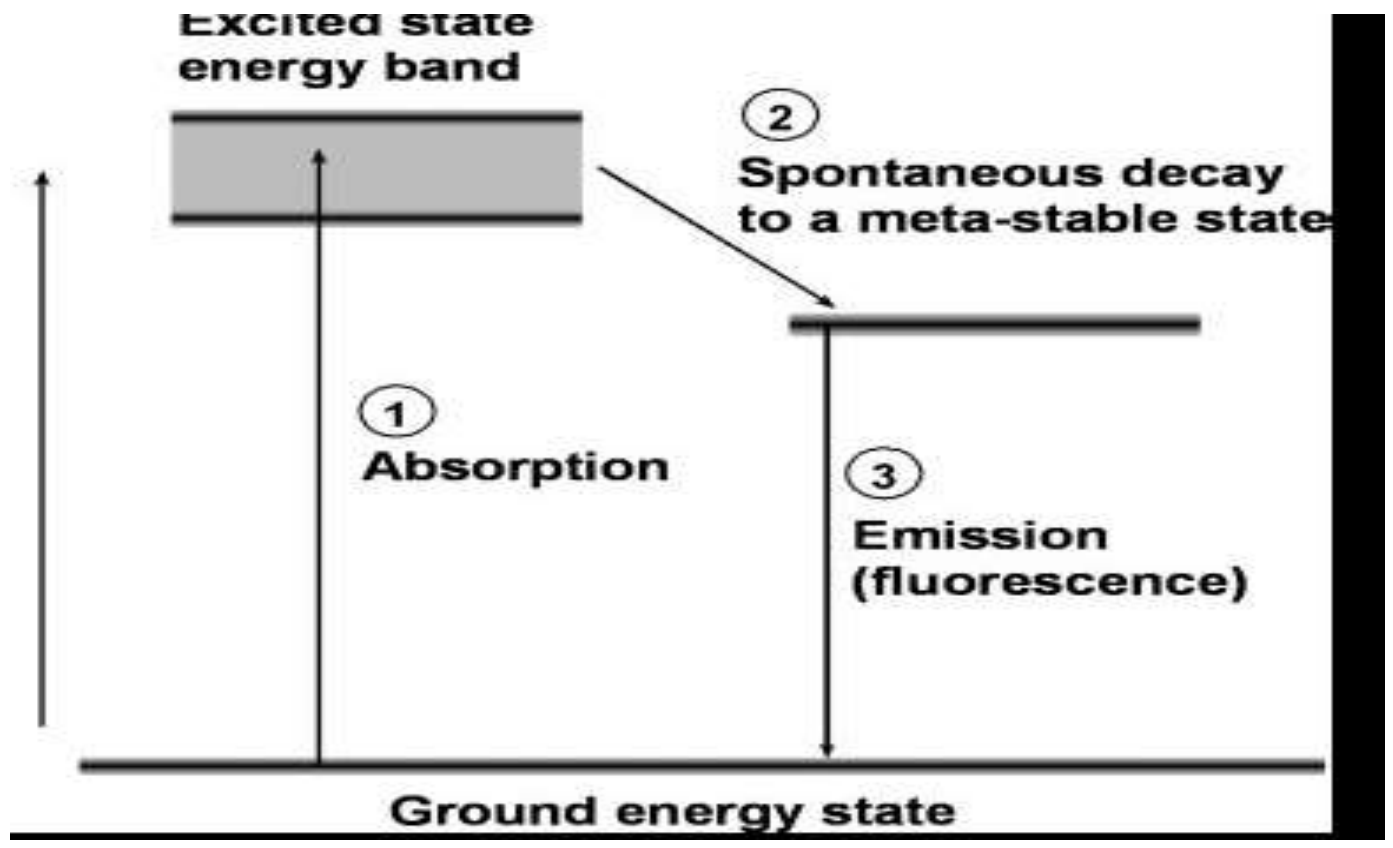

Fig. 1 A Basic Fluorescent Molecule's Energy-Level Illustration Steps (1). Electrons are excited by absorbing photon (2). Decaying of electrons (3). Electrons emits a photon with lower energy relative to the exciting photon

Reflected energy is measured with the spectrometer. Scientists can easily determine the amount of absorption by inspecting dips in the reflectance spectrum. Reflectance spectrum of Martian meteorite with absorption bands is shown as an example in Figure 2.

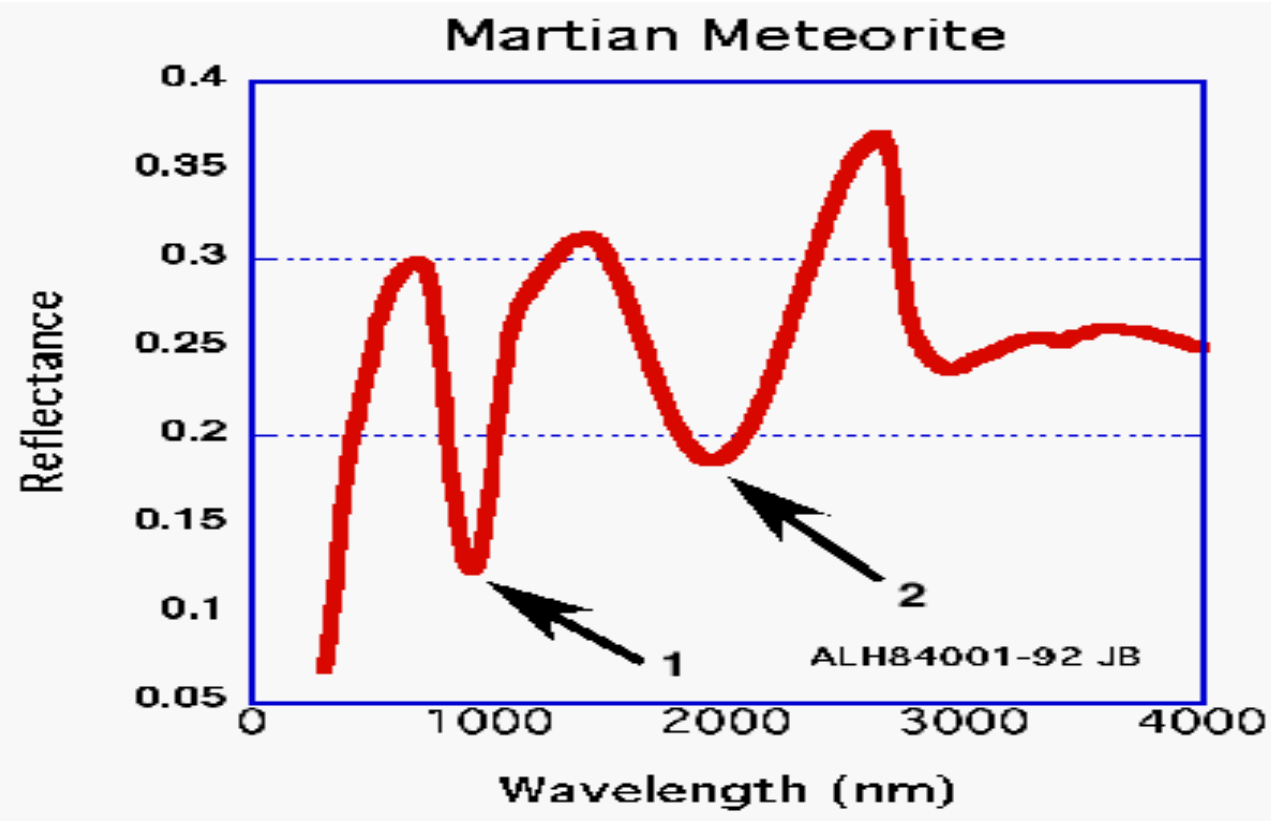

Fig. 2 Reflectance Spectrum of Martian Meteorite ALH84001 
The reflectance spectroscopy permit the scientists to observe where the light is being absorbed by electrons. In that spectrum at certain points where the light is being absorbed by electrons there will be falls in reflection. The properties of mineral composition are read by these absorption bands(dips) and are examined by gathering data about UV light and IR radiation in addition to the visible ranges. Spectrometer helps to estimate the chemical content of rock /soil of the Martian surface without analyzing actual rock samples. Once the spectra are obtained by automatically using existing tools like ENVI tool or manually by using algorithms then they are segmented by using other methods. Comparison of automatically retrieved spectra over the whole scene and manually selected spectra over the whole scene is depicted as an example in Figure 3.

Imaging spectroscopy in other words considered as hyper spectral imaging. It has been transformed into a commodity product available to a broad user community. At present, to deal with special attributes of hyper spectral data a standardized data processing techniques must be take into account[1].

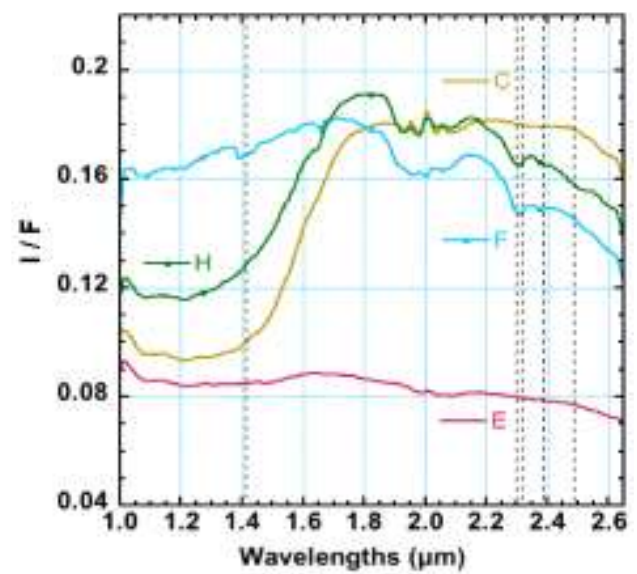

(a)

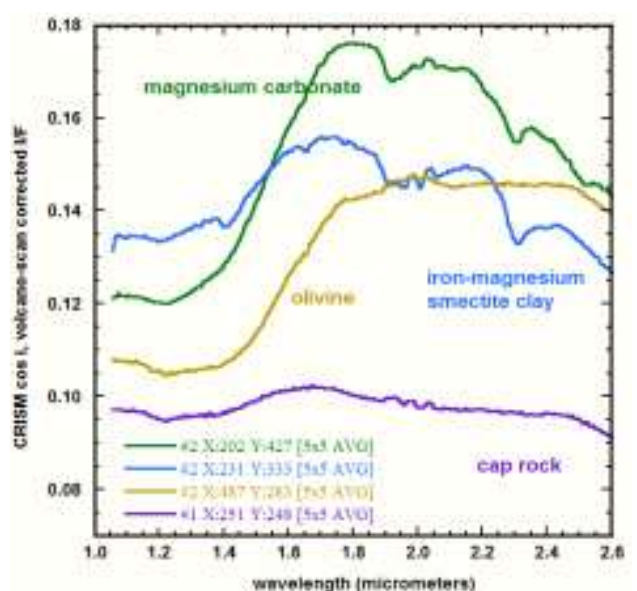

(b)

Fig. 3 Comparison of (a) Automatically retrieved spectra over the whole scene and (b) Manually selected spectra over the whole scene

As hyper spectral images contain a rich of data it becomes difficult to interpret and understand their properties. Further, it depends on the properties of ground materials that are trying to measure, and how they relate to the measurements actually made by the hyperspectral sensor. Hyperspectral sensors are important and powerful instruments for addressing classification problems in complex scenarios, as they allow one a elemental characterization of the spectral activities of the considered information classes. However, the processing of hyperspectral data is particularly complex both from a theoretical viewpoint [viz. problems related to the Hughes phenomenon (Hughes, 1968)] and from a computational perspective. In literature feature extraction and reduction methods have been explained by the different authors in hyperspectral data concept. Further, few authors are analysed and studied on the responsibility of spectral resolution on the classification accuracy in different application domains. The hyperspectral image analysis find several applications in target detection, material mapping, material identification and mapping details of surface properties etc. The hyperspectral image enhancement techniques reported in the literature are briefly summarized in the review of the related work.

The rest of the paper is organized into four sections: Section 2 reviews the developments in enhancement of the hyperspectral imagery to explore the spatial-spectral information. The proposed model of hyperspectral image enhancement by using various filters is described in Section 3. The experimental results obtained by employing ENVI 
tool and analysis of the results are given in Section 4. Finally the Section 5 concludes and enlist the future scope of the proposed work.

\section{REVIEW OF RELATED WORK}

In the literature several studies have focused on the selection of the optimal sets of hyper spectral channels for use in the classification phase. Of these many focused on the development of algorithms for the selection of the optimal features, given a certain classification problem[2]. In this context, the proposed paper can recall the feature selection algorithms based on a search strategy and a separability measure. The author Pudil et al., [3] discussed about common search strategies on hyperspectral data are the Sequential Forward Floating Selection[4]. The author Serpico \& Bruzzone, explained about the Steepest Ascent. As a separability measure, the authors Bhattacharyya and Djouadi et al., explained about distance measure.The Jeffries-Matusita distance by the author Bruzzone et al., as well as the transformed divergence distance by the author Richards \& Jia has contributed their work. The authors Backer et al., Schmidt \& Skidmore studied and analyzed the location of the most informative channels in the spectrum by considering the physical meaning of each band. Among them, proposed work recall the study of Backer et al., as they have analyzed different band selection methodologies and different spectral resolutions on a CASI 2 image acquired in 46 bands. The author Clark et al., explained how hyper spectral images have been used by geologists for mineral mapping [5]. The author Ben-Dor discussed how to detect soil properties including moisture, organic content, and salinity. The authors Clark et al., Aber and Martin and Merton have discussed about identification vegetation species, plant canopy chemistry and detection of vegetation stress [6, 7]. Military personnel have used hyperspectral imagery to detect military vehicles under partial vegetation canopy, and many other military target detection objectives $[8,9]$. The feature selection algorithms based on a search strategy and a separability measure. Most of the authors have found it difficult to extract the spatial and spectral features from the hyperspectral imagery acquired with spectroscopy, for effective end-member detection $[10,11]$. Hence there is scope to explore the different enhancement techniques in-order to explore the embedded spatial-spectral information in the hyperspectral imagery for better end-member detection and mineral classification of the Mars data. The detailed description of the proposed methodology is given in the next sections.

\section{PROPOSED METHODOLOGY}

In proposed work the input image is hyper-spectral imagery. This input hyper-spectral imagery is tested and compared with different filters using ENVI tool. Where the ENVI software helps to process the operations automated. ENVI software includes complete suite of hyper-spectral image analysis tool. ENVI software provides the easy and faster access of image from the server. For hyper spectral imagery, it is necessary to have filters for better quality. In proposed paper using ENVI tool different filters are applied on mars dataset and even compared. The input data image f970619t01p02_r02_sc01.a.rfl is considered from mars dataset [12]. In ENVI the filters use IDL routines for the enhancement purpose. MEDIAN(n) is a IDL routine for median filters, where [n] is referred as kernel size. The functional block schematic is shown in Figure 4.

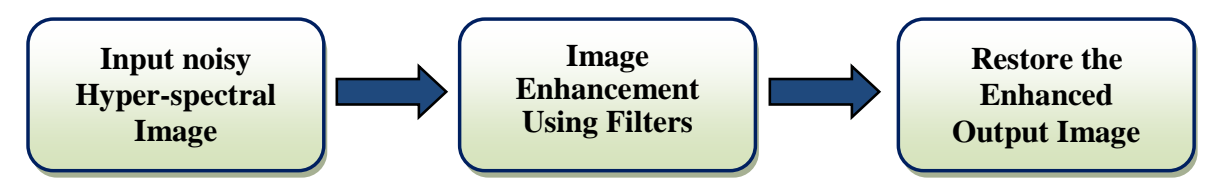

Fig. 4 Block Schematic of the Proposed Work for Hyperspectral Image Enhancement 
In order to extract the spatial spectral information of the hyperspectral image, it is required to enhance the hyperspectral image using filters for further processing. The choice of selecting the proper filter is important in the pre-processing step, as the improper selection of filter cause loss of precious information from the acquired image. Most of the authors have found it difficult to extract the spatial and spectral features from the hyperspectral imagery acquired with spectroscopy, for effective end-member detection. In the proposed work hyperspectral images are taken as input image from the spectral library from hyperspectral reflectance dataset of Mars dataset. The input Mars image dataset acquired by CRISM instrument contains various kinds of noisy information content [12, 13]. In the Mars image dataset different minerals such as iron oxides, hydrated minerals, surpentines and Olivines spectra. The iron oxides ranging from $0.9 \mu \mathrm{m}$ to $1.3 \mu \mathrm{m}$, the spectral characteristics of hydrated minerals at 1.4, 1.9 and $2.5 \mu \mathrm{m}$, powerful $\mathrm{M}$ shape duet absorptions of Surpentines spectra at the $1.91 \mathrm{am}$, spectral feature of Olivines spectra at 1 $\mu \mathrm{m}$ and other minerals spectral ranging from 0.2 to $2.02 \mu \mathrm{m}[14,15]$. In-order to explore these embedded spatial-spectral information in the hyperspectral imagery for further processing, it is required to pre-process viz. enhance the image. The selection of proper filter plays a vital role in enhancement, as the improper selection of the filter may leads to loss of precious information from the acquired image. Since the pixel resolution of the mars image is very large it requires the supportive software tools [16 - 20]. In the proposed work the ENVI tool provides different filters to enhance the image. Further the enhanced Mars image is stored for future processing steps.

\subsection{ENHANCEMENT OF HYPERSPECTRAL IMAGERY USING KERNELS}

In the proposed work, six different filters/kernels namely Sharpening, Smoothing, Median, Gaussian, Equalization and Square Root filters/kernels are employed to enhance the hyperspectral images in order to explore the embedded spatial-spectral information. The brief description of the filters employed in the proposed work is given in the following. Firstly the Sharpening Filter highlights/enhances the fine details of an image that has been blurred, either in error or as a natural effect at the time of image acquisition. The original image is filtered by a high-pass filter which extracts high-frequency components of the original image. A scaled version of the high-pass filter output is then added to the original image which produces a sharpened image. Sharpening process uses the kernel as a high pass filter to increases the contrast between bright and dark regions to bring out features. Further the Smoothing Filter produces a slow changes in value so that it is easier to see trends in the image data. Smoothing of an image is carried out to reduce noise within an image employing the low pass filters to produce a less pixelated image. The Median Filter is a nonlinear operation and is more effective than convolution [21]. It is often used in hyperspectral image preprocessing to reduce "salt and pepper" noise. Median filter helps to reduce noise and preserve edges. Further the Gaussian Filter is a linear filter usually used to blur the image or to reduce noise. The Gaussian Filter removes noise while keeping edges relatively sharp viz. blur edges and reduce contrast [22]. An equalization filter will self-adjusts the properties of time varying and also adopts transfer function according to an optimizing algorithm [23]. Finally the Square root Filter is used to process the uncorrelated observations with no process time [24]. If the dimension or the pixel resolution of the hyperspectral image is higher, then it will be more competitive type. In hyperspectral imaging, measuring the PSNR value for the enhanced image quality, Median filter and Gaussian filter are suitable for the effective pre-processing and to reduce noise. The Table I will summarize the functionalities of various filters employed in the proposed work. The next section depicts the experimental results of the proposed filters. 
Table I. Filter Employed for the Mars hyperspectral image Enhancement

\begin{tabular}{|c|l|l|}
\hline Sl.No. & \multicolumn{1}{|c|}{ Filter } & \multicolumn{1}{c|}{ Description } \\
\hline 1 & Sharpening Filter & $\begin{array}{l}\text { Increases the contrast between bright and dark regions } \\
\text { to bring out fine details of an image with a high pass } \\
\text { filter. }\end{array}$ \\
\hline 2 & Smoothing Filter & $\begin{array}{l}\text { Highlight the fine variations in pixel value to observe } \\
\text { trends in the image data and to produce a less pixelated } \\
\text { image employing the low pass filter. }\end{array}$ \\
\hline 3 & Median Filter & $\begin{array}{l}\text { Reduce noise and preserve edges with a nonlinear } \\
\text { operation to reduce "salt and pepper" noise. }\end{array}$ \\
\hline 5 & Gaussian Filter & $\begin{array}{l}\text { Removes noise while keeping edges relatively sharp } \\
\text { viz. blur edges and reduce contrast with a linear filter } \\
\text { operation to blur the image or to reduce noise. }\end{array}$ \\
\hline 6 & Square root Filter & $\begin{array}{l}\text { Self-adjusts the properties of time varying and also } \\
\text { adopts transfer function according to an optimizing } \\
\text { algorithm. } \\
\text { time. }\end{array}$ \\
\hline
\end{tabular}

\section{EXPERIMENTAL ANALYSIS}

In the proposed work input data image f970619t01p02_r02_sc01.a.rfl [11,14] is considered from Mars dataset for experimentation. For the input image RGB bands are selected to enhance the image, as R.Band-7,G.Band-9 and B.Band-11. The mentioned band values are derived from the spectral profile of f970619t01p02_r02_sc01.a.rfl as shown in the Figure 5.

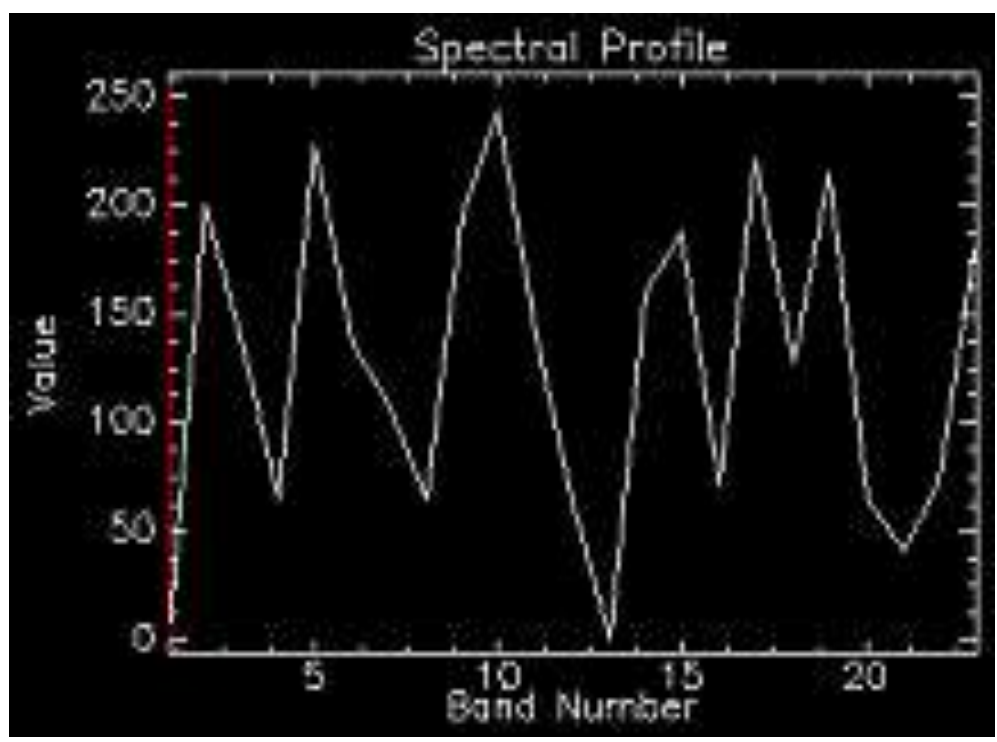

Fig. 5 Spectral profile of f970619t01p02_r02_sc01.a.rfl

In the proposed work input Mars image is depicted in Figure 6(a). Further the input image is enhanced with sharpen filter which is shown in Figure 6(b).Which is necessary when researcher is concentrating on geospatial information. Next in Figure 6(c) the input image is enhanced using smoothing filter, this is also done for the geospatial information. The filters used in Figure 6(d) to Figure 6(g) are helpful for the fetching information like spatial and spectral end-members. Comparing each filters with one other it is concluded that each one is having its own applications in respective area. But Gaussian filter and 
Median filters play a vital role in especially in finding end-members of mineral composition present in mars surface.

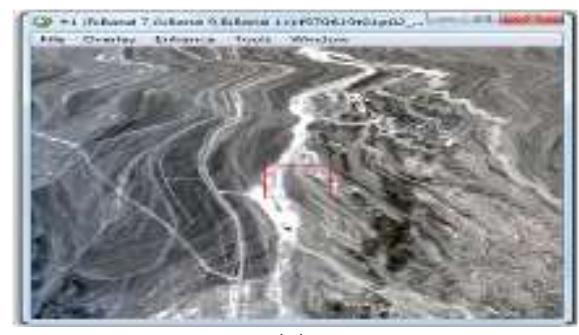

(a)



(b)

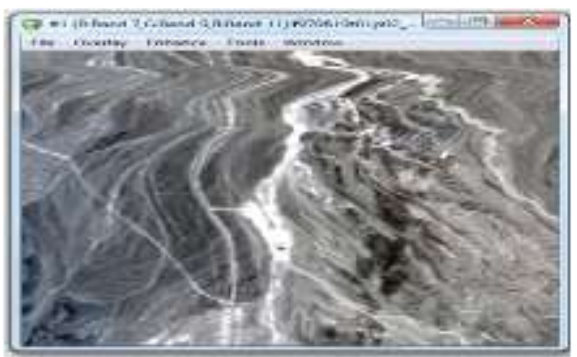

(d)

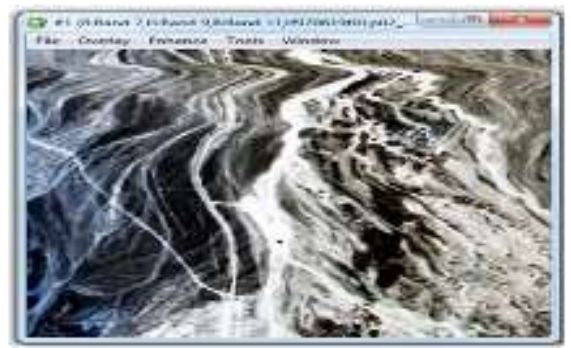

(f)

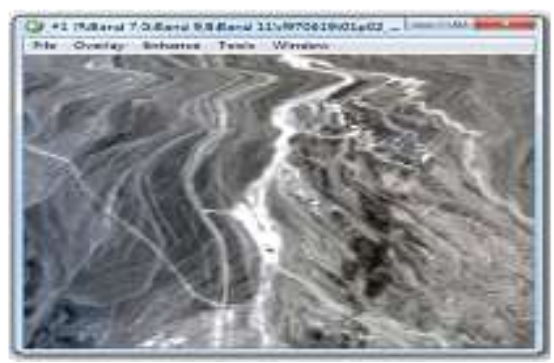

(c)

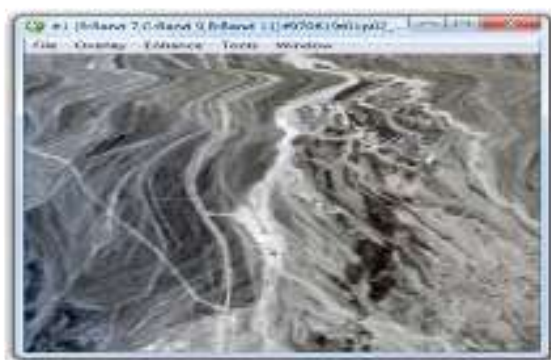

(e)

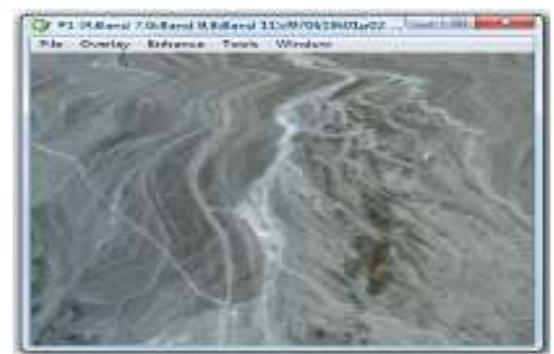

(g)

Fig. 6. (a) Original Image (b) Sharpen Filter Image (c) Smooth Filter Image (d) Median Filter Image (e) Gaussian Filter Image (f) Equalization Filter Image (g) Square Root Filter Image

\section{CONCLUSION}

The proposed work reveals that, in order to explore end-members out of the spatialspectral information it is necessary to choose better enhancement algorithm (filters/kernels). The experimentation is carried out by employing six variety of filters/kernels namely Sharpening Filter, Smoothing Filter, Median Filter, Gaussian Filter, Equalization Filter and Square root Filter. Further the PSNR values are computed to verify the spatial information quality of enhanced hypersepectral image. Median and Gaussian 
kernels gives better enhanced image compared to other mentioned filters. Especially for finding mineral composition of mars surface Median and Gaussian filters are helpful. In future the experimentation is to be extended on different Mars dataset to find the optimal kernel/filter or hybrid filters for better enhancement of the hyperspectral imagery in order to identify the mineral composition. Further explore active learning/deep learning approach to enhance the performance of the identification system for different mineral composition by employing enhanced version of the hyperspectral image.

\section{ACKNOWLEDGMENT}

We would like to express our thanks to all the researchers who have contributed to the domain of hyper spectral image analysis. We wish to express our deepest gratitude and warmest appreciation to all the authors of published articles.

\section{REFERENCES}

[1] Shippert P., "Why Use Hyperspectral Imagery," Photogrammetric Engineering \& Remote Sensing, (2004), pp. 377-380.

[2] Keshava N., Kerekes J., Manolakis D., Shaw G., "An Algorithm Taxonomy for Hyperspectral Unmixing", Algorithms for Multispectral, Hyperspectral, and Ultraspectral imagery VI, Proceedings of SPIE, vol. 4049, (2000), pp. 42-63.

[3] Pudil et al., "Floating Search Methods in Feature Selection," Pattern Recognition Letters, Vol.15, (1994), pp. 1119-1125.

[4] Plaza A., Martínez P., Pérez R., and J. Plaza, "A Quantitative and Comparative Analysis of Endmember Extraction Algorithms From Hyperspectral Data," IEEE Transactions on Geo-Science and Remote Sensing, Vol. 42, No. 3, (2004), pp. 650-663.

[5] Mario Parente, Antonio Plaza. "Survey of Geometric and Statistical Unmixing Algorithms for Hyperspectral Images", Second Workshop on Hyperspectral Image and Signal Processing:Evolution in Remote Sensing, (2010).

[6] Dalponte, M.. "The Role of Spectral Resolutionand Classifier Complexity in the Analysis of Hyperspectral Images of Forest Areas", Remote Sensing of Environment, (2009), pp. 11-16.

[7] Yuval Garini"Spectral Imaging: Principles and Applications", International Society for Analytical Cytology Cytometry Part A 69A:pp. 735-747, (2006).

[8] CRISM Hyperspectral Imagery, www. crism.jhuapl.edu.

[9] Pawan Kumar Patidar, "Image Filtering using Linear and Non Linear Filter for Gaussian Noise", International Journal of Computer Applications (0975 - 8887), Vol. 93, No.8, (2014).

[10] Fatemeh Kowkabi, Hassan Ghassemian, Ahmad Keshavarz,"Enhancing Hyperspectral Endmember Extraction Using Clustering and Oversegmentation-Based Preprocessing," IEEE Journal of Selected Topics in Applied Earth Observations and Remote Sensing, Vol.9, Issue 6, (2016), pp. 2400-2413.

[11] Olivier Eches, Nicolas Dobigeon and Jean-Yves Tourneret, "Enhancing Hyperspectral Image Unmixing with Spatial Correlations", IEEE Transactions on Geoscience and Remote Sensing,Vol. 49, Issue 11,(2012), pp. 4239-4247.

[12] E.Millour," The Latest (Version 4.3) Mars Climate Database",Mars Atmosphere: Modeling and Observations (2008).

[13] Rashmi Karchi and Nagesh B.K,“A Review of Spectral Unmixing Algorithms in the Context of Mars Dataset", International Journal of Latest Trends in Engineering and Technology (IJLTET), ISSN:2278621X, (2013), pp. 55-60.

[14] Nagarajan Munusamy and Rashmi. P. Karchi, "Hyperspectral Image Classification and Unmixing by using ART and SUnSPI Techniques", International Journal of Recent Technology and Engineering (IJRTE), Volume 8, Issue 1, (2019), pp. 777-784.

[15] Nagarajan Munusamy and Rashmi. P. Karchi, "Exploration of Unmixing and Classification of Hyperspectral Imagery ", International Journal of Innovative Technology and Exploring Engineering (IJITEE), Volume 8, Issue 7, (2019), pp. 723-733.

[16] M. Ezhilarasi V. Krishnaveni "A Survey on Wireless Sensor Network: Energy and Lifetime Perspective" Taga Journal vol. 14, pp. 3099-3113 ISSN 1748-0345.

[17] Ezhilarasi, M. \& Krishnaveni, V. "An evolutionary multipath energy-efficient routing protocol (EMEER) for network lifetime enhancement in wireless sensor networks" Soft Computing, (2019). https://doi.org/10.1007/s00500-019-03928-1.

[18] M. Ezhilarasi and V. Krishnaveni, "A Survey on Energy and Lifetime in Wireless Sensor Networks", International Journal of Soft Computing, (2018), Vol. 13, pp. 167-182. 
[19] M. Nagarajan and S. Karthikeyan, "A New Approach to Increase the Life Time and Efficiency of Wireless Sensor Network", IEEE International Conference on Pattern Recognition, Informatics and Medical Engineering (PRIME), (2012), pp. 231-235.

[20] Nagarajan Munusamy, Karthik Srinivasan , " Various Node Deployment Strategies in Wireless Sensor Network " , IPASJ International Journal of Computer Science(IIJCS), Volume 5, Issue 8, August (2017), pp. 039-044 , ISSN 2321-5992.

[21] S. S. Wali, S. M. Hatture and S. Nandyal, "MFCC Based Text-Dependent Speaker Identification Using BPNN", International Journal of Signal Processing Systems Vol. 3, No. 1, (2015), pp. 30-34.

[22] Nalinakshi B. G, Sanjeevakumar M. Hatture, Manjunath S.Gabasavalgi, Rashmi P. Karchi, "Liveness Detection Technique for Prevention of Spoof Attack in Face Recognition System ", International Journal of Emerging Technology and Advanced Engineering, Vol. 3, Issue 12, (2013), pp. 627-633.

[23] Shanmukhappa A Angadi, Sanjeevakumar M Hatture,"Face Recognition Through Symbolic Modeling of Face Graphs and Texture", International Journal of Pattern Recognition and Artificial Intelligence, Vol. 33, Issue 12, (2019), 23 pages.

[24] Sanjeevakumar M. Hatture, Susen P. Naik, "Agro Guardian: A Smart Agriculture Framework for Precision Farming", Modern Techniques for Agricultural Disease Management and Crop Yield Prediction, IGI Global, (2020), pp. 179-202. 
International Journal of Advanced Science and Technology Vol.136 (2020) 